\title{
THE DESIGN OF A CLASS OF PREFECT RECONSTRUCTION TWO-CHANNEL FIR AND WAVELETS FILTERBANKS USING CONSTRAINED LEAST SQUARES METHOD AND SEMIDEFINITE PROGRAMMING
}

\author{
S. C. Chan, Carson K. S. Pun and K. L. Ho

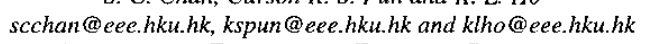 \\ Department of Electrical and Electronic Engineering \\ The University of Hong Kong, Pokfulam Road, Hong Kong
}

ABSTRACT

This paper proposes two new methods for designing a class of 2-channel PR FIR filterbanks and wavelets with $K$ regularity of high order. The $K$-regularity constraints are expressed as a set of linear constraints in the design variables. The first method formulates the design problem as a quadratic programming problem with linear equality constraints (QPLC), which can be solved using the method of Lagrange multiplier. The second design method employs the minimax error criteria and solves the design problem as a semidefinite programming problem (SDP). By removing the redundant variables, the equality constraints are automatically imposed into the design problem. The optimization problem is then formulated as a linear convex objective function subject to a union of affine set which can be represented by a set of linear matrix inequalities. Hence they can be solved using existing SDP solver. Design examples are given to demonstrate the effectiveness of the proposed methods.

\section{INTRODUCTION ,}

Perfect reconstruction (PR) multirate filterbanks (FB) have important applications in signal analysis, signal coding and the design of wavelet bases. A number of techniques for designing linear-phase and low-delay twochannel PR FIR filter banks are now available [1-11]. In [2], it was shown that the structurally PR 2-channel FB proposed in [1] can be formulated as a complex Chebyshev approximation problem, which can be solved using the REMEZ exchange algorithm to obtain linearphase as well as low-delay FBs. The construction of wavelet basis from these FBs satisfying certain $K$ regularity condition up to one zero order moment was also studied. The advantage of these FBs is their low implementation and arithmetic complexities. The incorporation of $K$-regularity with higher order, however, was not addressed due to the difficulties in solving the constrained optimization problem. The design of paraunitary 2-channel FBs (the conjugate quadrature filters (CQF)) with prescribed number of $K$-regularity was previously considered in [3]. The FBs (wavelets) so obtained are in general not linear-phase (antisymmetric). Recently, Tay [10] showed that it is possible to incorporate the $K$-regularity condition (or equivalently a certain number of zeros of the lowpass analysis filter and highpass analysis filter at $\omega=0$ and $\omega=\pi$ respectively) for the linear-phase FB in [1] using the Berstein polynomial. Since the error function is a linear function of the coefficients in the Berstein polynomial, it can be solved as a simple quadratic programming problem if the least squares error criteria is used. The Genetic Algorithm (GA) was also proposed for designing such FBs and wavelets with higher order moments and sumof-powers-of-two (SOPOT) coefficients [8].

In this paper, we extend the approach in [2] to the case of higher order $K$-regularities. This will enable a larger class of wavelet FBs with different smoothness and delay to be constructed. More precisely, we show that it is possible to formulate the design problem as a quadratic programming problem with linear constraints (QPLC), if a least squares criteria is employed. The approach is rather general and it applies to the linearphase as well as the low-delay cases without making use of the Berstein polynomial expansion. We also show that for the minimax error criteria, the design problem can be solved using semidefinite programming (SDP) [15]. SDP has been successfully applied in areas, such as control, logistic, digital filter design [6], digital signal processing, etc. Thanks to the interior-point method [1214], SDP can now be solved efficiently in polynomial time. Design results show that the proposed constrained least squares design method gives rise to least squares optimal solution that satisfies the required $K$-regularity condition, whereas the SDP design method produces filters with almost equi-ripple approximation error while satisfying the given regularity condition. Comparing the result reported in [2] for $K=1$, considerable improvement was achieved using the SDP approach for the same design specification.

The paper is organized as follows: In section 2, a brief description of the FB to be designed and the problem formulation will be given. Section 3 is devoted to the proposed constrained least squares design method. The second design method using the SDP will be introduced in Section 4. This is followed by several design examples in Section 5. Finally, conclusions are drawn in section 6 .

\section{Problem Formulation}

The structurally PR FBs $[1,2]$ that we are going to design is shown in figure 1. It can be seen that the FB is parameterized by the sub-filter pairs $\beta(z)$ and $\alpha(z)$ and the delay parameters $N$ and $M$. The FB is PR for arbitrary choice of filter pairs $\beta(z)$ and $\alpha(z)$. The functions $\alpha(z)$ and $\beta(z)$ can be chosen as linear-phase FIR or all-pass functions to realize FIR and IIR filter banks with very low design and implementation complexities. In [1], the case of using identical $\alpha(z)$ and $\beta(z)$ with delay parameter $M=2 N-1$ is studied so as to obtain linear-phase FIR or passband linear-phase IIR FBs. Later in [2], $\alpha(z)$ and $\beta(z)$ are generalized to include general FIR functions. As the linear-phase requirement is relaxed, the lengths of $\alpha(z)$ and $\beta(z)$ are no longer restricted by the delay parameters of the system. Therefore, higher stopband attenuation can still be achieved at low system delay. In addition, wavelets FBs with one zero order moment was constructed. 
Imposing $K$-regularity of higher order generally requires the solution of a constrained optimization problem, as we shall see lar in Sections 3 and 4.

From 1 . 1 . the frequency responses of the analysis filters are given $t \cdot y$ :

$$
\begin{aligned}
& H_{0}\left(e^{j \omega}\right)=\frac{1}{2}\left(e^{-j 2 N \omega}+e^{-j \omega} \beta\left(e^{j 2 \omega}\right)\right) \\
& H_{1}\left(e^{j \omega}\right)=-\alpha\left(e^{j 2 \omega}\right) H_{0}\left(e^{j \omega}\right) \div e^{j(-2 M-1) \omega}
\end{aligned} .
$$

Let $L_{\beta}$ and $L_{\alpha \alpha}$ be respectively the lengths of $\beta\left(e^{j \omega}\right)$ and $\alpha\left(e^{j \omega}\right)$. From [2], it v'as shown that the desired responses of $\beta\left(e^{j \omega}\right)$ and $\alpha\left(e^{j \omega}\right)$ are respectively

$$
\begin{aligned}
& \beta_{d}\left(e^{j \omega}\right)=e^{j(-N+1 / 2) \omega \omega}, \\
& \text { and } \alpha_{d}\left(e^{j \omega}\right)=e^{j(-M+N-1 / z) \omega}, \quad \omega \in[-\pi, \pi]
\end{aligned}
$$

Next, we shall show that the $K$-regularity can be written as a set of linear constraints in the coefficients of $\beta\left(e^{j \omega t}\right)$ and $\alpha\left(e^{j \omega}\right)$, and the design problem can be solved using a QPLC or a SDP problem.

\section{Constrained Least SQuares Design}

The objective functions for the least squares design of $\beta\left(e^{j \omega}\right)$ and $\alpha\left(e^{j \omega}\right)$ are

$$
\begin{aligned}
& L_{2}(\beta)=\int_{-2 \omega_{\beta_{p}}}^{22 \omega_{\beta}} W(\omega)\left|\boldsymbol{\beta}^{T} \cdot \boldsymbol{e}_{L_{\beta}}-\beta_{d}\left(e^{j \omega}\right)\right|^{2} d \omega \\
& L_{2}(\alpha)=\int_{-2 \omega_{\psi}}^{2 \omega_{\omega}} W(\omega)\left|\boldsymbol{a}^{T} \cdot \boldsymbol{e}_{L_{u}}-\alpha_{d}\left(e^{j \omega}\right)\right|^{2} d \omega
\end{aligned}
$$

where $e_{q}=\left[\begin{array}{llll}1 & e^{-j \omega} & \cdots & e^{-j(q-1) \omega}\end{array}\right]^{T} \cdot \omega_{\beta p}$ and $\omega_{\alpha p}$ are the passband cutoff frequencies of $H_{0}\left(e^{j \omega}\right)$ and $H_{1}\left(e^{j \omega}\right)$ respectively. $\quad \beta=\left[\begin{array}{lllll}\beta_{0} & \beta_{1} & \cdots & \beta_{t_{\mathrm{A}-1}-1}\end{array}\right]^{T} \quad$ and $\alpha=\left[\begin{array}{llll}\alpha_{0} & \alpha_{1} & \cdots & \alpha_{r_{z-1}}\end{array}\right]^{T}$ are the impulse responses of $\beta(z)$ and $\alpha(z)$ respectively, and $W(\omega)$ is a positive weighting function. Expanding equation (3a), we have

$$
\begin{aligned}
& L_{2}(\beta)=\boldsymbol{\beta}^{T} \cdot \boldsymbol{W}_{\beta} \cdot \boldsymbol{\beta}-2 \boldsymbol{\beta}^{T} \cdot \boldsymbol{g}_{\beta}+p_{\beta} \\
& L_{2}(\alpha)=\boldsymbol{\alpha}^{T} \cdot \boldsymbol{W}_{\alpha} \cdot \boldsymbol{\alpha}-2 \boldsymbol{\alpha}^{T} \cdot \boldsymbol{g}_{\alpha}+p_{\alpha}
\end{aligned}
$$

where

$$
W_{i}=\int_{-2 \omega_{i p}}^{2 \omega_{i p}} W(\omega) e_{l_{i}} \cdot e_{h_{i}}^{H} d \omega
$$

$g_{i}=\int_{-2 \omega_{i p}}^{2 \omega_{i p}} \operatorname{Re}\left(W(\omega) \boldsymbol{e}_{L_{i}} \cdot i_{j}^{*}\left(e^{j \omega}\right)\right) d \omega$ and $\boldsymbol{p}_{i}$ are sôme constants with $i=\alpha, \beta$. The optimal least squares solution is obtained by differentiating (3b) with respect to the optimization vector variables and setting the resulting derivatives to zero. It gives

$$
\beta_{o p t}=W_{\beta}^{-1} \cdot g_{\beta} \text { and } \alpha_{p p t}=W_{\alpha}^{-1} \cdot g_{\alpha}
$$

To construct a wavelet FBs, the analysis filters $H_{0}\left(e^{j \omega}\right)$ and $H_{1}\left(e^{j \omega}\right)$ should possess at least one zero at $\omega=\pi$ and $\omega=0$, respectively. A higher order of $K$-regularity can be obtained by imposing $K_{0}$ and $K_{1}$ zeros at $\omega=\pi$ and $\omega=0$ for $H_{0}\left(e^{j \omega}\right)$ and $H_{1}\left(e^{j \omega}\right)$, respectively (with $\left.K_{0} \geq K_{1}>1\right)$. This is equivalent to the following

$$
\left[\frac{\partial^{k_{0}}}{\partial \omega^{k_{0}}} H_{0}\left(e^{j \omega}\right)\right]_{\omega=\kappa}=\left[\frac{\partial^{k_{1}}}{\partial \omega^{k_{1}}} H_{1}\left(e^{j \omega}\right)\right]_{\omega=0}=0, \text {, (5) }
$$

for $k_{0}=0, \cdots, K_{0}-1$ and $k_{1}=0, \cdots, K_{1}-1$. For incorporating the regularity constraints for $H_{1}\left(e^{j \omega}\right)$, we have to assume the regularity constraint is already incorporated in $H_{0}\left(e^{j \omega}\right)$. After performing the differentiation, the regularity constraints of (5) can be expressed in terms of the coefficients of $\beta(z)$ and $\alpha(z)$ as follows:

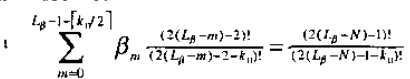

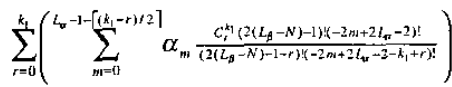

$$
\begin{aligned}
& =\frac{\left(2\left(L_{\theta}+L_{\beta}-M\right)-4\right) !}{\left(2\left(L_{u}+L_{B}-M\right)-4-k_{1}\right) !},
\end{aligned}
$$

for $k_{0}=0, \cdots K_{0}-1$ and $k_{1}=0, \cdots K_{1}-1$. Obviously, (6a) is a linear combination of the optimization parameters with constant coefficients, which represents a set of linear constraints to be satisfied by $\beta$ and $\alpha$. (6a) can also be written more compactly in matrix form as

$$
\boldsymbol{B} \cdot \boldsymbol{\beta}=\boldsymbol{c}_{\beta, \mathrm{K}_{0}} \text { and } \boldsymbol{A} \cdot \boldsymbol{\alpha}=\boldsymbol{c}_{\alpha, K_{\mathrm{t}}}
$$

for some matrices $\boldsymbol{B}$ and $\boldsymbol{A}$, and vectors $\boldsymbol{c}_{\beta, K_{\mathrm{V}}}$ and $\boldsymbol{c}_{r, K_{1}}$, whose entries are defined in (6a). (3a) and (6a) together form a quadratic programming problem subject to a set of linear equality constraints, which can be solved analytically using the method of Lagrange multiplier [16]. Define the following Lagrangean functions

$$
\begin{aligned}
\tilde{L}_{2}(\boldsymbol{\beta}) & =\boldsymbol{\beta}^{T} \cdot \boldsymbol{W}_{\beta} \cdot \boldsymbol{\beta}-2 \boldsymbol{\beta}^{T} \cdot \boldsymbol{g}_{\beta}+\boldsymbol{p}_{\beta} \\
& +\lambda_{\beta}^{T} \cdot\left(\boldsymbol{B} \cdot \boldsymbol{\beta}-\boldsymbol{c}_{\beta, K_{\mathrm{v}}}\right), \\
\tilde{L}_{2}(\boldsymbol{\alpha}) & =\boldsymbol{\alpha}^{T} \cdot \boldsymbol{W}_{\alpha} \cdot \boldsymbol{\alpha}-2 \alpha^{T} \cdot \boldsymbol{g}_{\alpha}+p_{\alpha} \\
& +\lambda_{\alpha}^{T} \cdot\left(\boldsymbol{A} \cdot \boldsymbol{\alpha}-\boldsymbol{c}_{\alpha \cdot K_{1}}\right),
\end{aligned}
$$

where $\lambda_{\beta}$ and $\lambda_{\alpha}$ are the Lagrange multipliers associated with $\beta$ and $\alpha$, respectively. Differentiate (7) with respect to the corresponding vector variables, we have

$$
\begin{aligned}
& \beta_{o p t}=W_{\beta}^{-1} \cdot g_{\beta}-\frac{1}{2} W_{\beta}^{-1} \cdot \hat{\lambda}_{\beta}^{T} \cdot B \\
& \alpha_{a p t r}=W_{\alpha}^{-1} \cdot g_{\alpha}-\frac{1}{2} W_{\alpha}^{-1} \cdot \lambda_{\alpha}^{T} \cdot A .
\end{aligned}
$$

Si.7ce equation (8a) has to satisfy (6b), the Lagrange multipliers are determined to be

$$
\begin{aligned}
& \lambda_{\beta}=2\left(B B^{T}\right)^{-1}\left(g_{\beta}-W_{\beta}\left(B^{T} B\right)^{-1} B^{T} c_{\beta, k_{\alpha}}\right)^{T}, \\
& \lambda_{\alpha}=2\left(A A^{T}\right)^{-1}\left(g_{\alpha}-W_{\alpha}\left(A^{T} \boldsymbol{A}\right)^{-1} A^{T} c_{\alpha, k_{1}}\right)^{T} .
\end{aligned}
$$

Substituting equation $(8 \mathrm{~b})$ into $(8 \mathrm{a})$, we obtain the final desired solution.

\section{Semidefinite Programming (SDP) Design}

For the case of minimax design criteria with a prescribed number of zeros, we resort to SDP. For brevity of presentation, only the SDP design of $\beta\left(e^{j \omega}\right)$ will be described. The design of $\alpha\left(e^{j \omega}\right)$ is similar. First of all, minimizing the maximum ripple of the approximation error is equivalent to the following

$$
\min _{\beta}\left|\boldsymbol{\beta}^{T} \cdot e-\beta_{d}\left(e^{j \omega}\right)\right|^{2}=\alpha_{k}^{2}(\omega)+\alpha_{i}^{2}(\omega) \leq \delta
$$

for $\omega \in\left[0,2 \omega_{\beta p}\right]$, where

$$
\begin{aligned}
& \alpha_{R}(\omega)=\beta^{T} \cdot c-\operatorname{Re}\left(\beta_{d}\left(e^{j \omega}\right)\right) \\
& \alpha_{i}(\omega)=\beta^{T} \cdot s+\operatorname{Im}\left(\beta_{d}\left(e^{j \omega}\right)\right) .
\end{aligned}
$$

To minimize the metric in (9a) using SDP, we densely discretize $\omega$ over the band of interest $\omega \in\left[0,2 \omega_{\beta p}\right]$ into a 
set of frequencies points $\omega_{k}$ 's, $k=1, \cdots K .(9 \mathrm{a})$ is then approximated as:

$$
\begin{aligned}
& \min \delta \\
& \alpha_{k}^{2}\left(\omega_{k}\right)+\alpha_{i}^{2}\left(\omega_{k}\right) \leq \delta
\end{aligned} \quad k=1, \cdots K
$$

Using Schur complement [15], it can be shown that [6] (10a) is equivalent to

$$
\begin{aligned}
& \min \delta \\
& F_{k}(h) \geq 0 \quad k=1, \cdots K .
\end{aligned}
$$

where $F_{k}(h)=\left(\begin{array}{ccc}\delta & \alpha_{R}\left(\omega_{k}\right) & \alpha_{i}\left(\omega_{k}\right) \\ \alpha_{R}\left(\omega_{k}\right) & 1 & 0 \\ \alpha_{i}\left(\omega_{k}\right) & 0 & 1\end{array}\right)$. Since $F_{k}(h)$ is

affine in $\beta$, it is equivalent to a set of linear matrix inequalities (LMI). Define the augmented variable $x^{T}=\left[\begin{array}{ll}\delta & \beta^{T}\end{array}\right]$. The optimization problem in (10b) can be cast into the following standard LMI or SDP optimization problem

$$
\begin{aligned}
& \min c^{T} \cdot x \\
& F(x) \geq 0
\end{aligned},
$$

where $F(x)=\operatorname{diag}\left(F_{1}(x), F_{2}(x), \cdots F_{K}(x)\right)$. Theoretically, it is possible to determine whether a feasible solution exists for a SDP such as (10c), and if so, it is possible to determine the global optimal solution, since the problem is convex. In order to simultaneously solve the SDP problem (10c) and the regularity constraints (6b), the dependent variables defined by the equality constraints (6b) are expressed as a linear combination of the independent variables. The number of optimization variables is therefore reduced. It not only speeds up the optimization process but also structurally imposes the regularity condition. To remove the redundant optimization variables, we can rewrite (6b) as follows:

$$
\left[\begin{array}{ll}
\boldsymbol{B}_{n-r} & \boldsymbol{B}_{r}
\end{array}\right] \cdot\left[\begin{array}{c}
\beta_{n-r} \\
\beta_{r}
\end{array}\right]=c_{\beta \cdot k_{0}}
$$

where $\boldsymbol{B}=\left[\begin{array}{ll}\boldsymbol{B}_{n-r} & \boldsymbol{B}_{r}\end{array}\right], \boldsymbol{\beta}=\left[\begin{array}{c}\boldsymbol{\beta}_{n-r} \\ \boldsymbol{\beta}_{r}\end{array}\right]$, and the subscripts $r$ and $n-r$ indicate the redundant and non-redundant parts, respectively. Using equation (11), $\beta$ can be written in terms of $\beta_{n-r}$ as:

$$
\beta=\left[\begin{array}{c}
0 \cdot \boldsymbol{I}_{n-r} \\
\boldsymbol{B}_{r}^{-i} c_{\beta, k_{0}}
\end{array}\right]+\left[\begin{array}{c}
\boldsymbol{I}_{n-r} \\
-\boldsymbol{B}_{r}^{-1} \boldsymbol{B}_{n-r}
\end{array}\right] \boldsymbol{\beta}_{n-r} .
$$

Substituting (12) into (10c) and redefine $x^{T}=\left[\begin{array}{ll}\delta & \beta_{n-r}^{T}\end{array}\right]$, we still have the objective function and the constraints affine in $x$. In other words, it is still a standard SDP problem. The design of $\alpha(z)$ is similar to that of $\beta(z)$, and is omitted here for simplicity.

\section{DESIGN EXAMPLES}

For comparison purpose, a 2-channel PR FBs with the same specification as example 4.1 in [2] is designed using the proposed QPLC and the SDP methods. However, the number of zeros to be imposed for $H_{0}\left(e^{j \omega}\right)$ and $H_{1}\left(e^{j \omega}\right)$ are $K_{0}=2$ and $K_{1}=1$, respectively. The lengths of $\beta(z)$ and $\alpha(z)$ are $L_{\beta}=8$, and $L_{\alpha}=10$, respectively. The delay parameters are $N=2$ and $M=1$. Since $L_{\beta} / 2-N=2$ and $L_{\alpha} / 2-M=4$, it is a low-delay FB. The passband cutoff frequencies are $\omega_{\beta_{p}}=0.34 \pi$ and $\omega_{\alpha p}=0.66 \pi$, respectively. The results for the least squares-based method are shown in figure 2 .

For the SDP design method, $\omega$ in the passband is uniformly discretized using 30 samples. The optimization is carried out using the MATLAB LMI Toolbox and it takes less than 40 iterations to obtain the solution. The design results are shown in figure 3 . It can be seen that the stopband attenuation of $H_{0}(z)$ is $41.5 d B$ while that of [2] is a bit less than $40 d B$. As for $H_{1}(z)$, our design gives a stopband attenuation of $46.3 d B$, which is $6 d B$ better than that in [2].

\section{CONCLUSIONS}

We have presented 2 methods for designing a class of 2 channel PR FiR FBs and wavelets previously proposed in $[1,2]$. The $K$-regularity conditions are expressed as a set of linear equality conditions and the design problem is formulated as either a QPLC or a SDP problem depending on whether the least squares and minimax design criterion are used respectively. Design results show that FBs and wavelets with good quality and high order of $K$-regularity can be designed by the proposed methods.

\section{REFERENCES}

[1] S. M. Phoong, C. W. Kim, P.P. Vaidyanathan and R. Ansari, "A New Class of Two-Channel biothogonal Filter Banks and Wavelet Bases," IEEE Trans. SP, vol. 43, pp.649-664, Mar. 1995.

[2] J. S. Mao, S. C. Chan, W. Liu and K. L. Ho, "Design and multiplier-less implementation of a class of two-channel PR FIR filterbanks and wavelets with low system delay," IEEE Trans. $S P$, vol. 48, pp. 3379-3394, Dec 2000.

[3] P. Duhamel and O. Rioul, "A Remez exchange algorithm for orthonormal wavelets," IEEE Trans. CAS II, vol. 41, pp. 550-560, Aug. 1994.

[4] G. Schuller and M. J. T. Smith, "A new algorithm for efficient low delay filter bank design," in Proc. IEEE ICASSP'95, vol. 2, pp. 1472 - 1475, May. 1995.

[5] R. Gandhi and S. K. Mitra, "Desion of two-channel low delay perfect reconstruction filter banks," in Proc. $32^{\text {nd }}$ International Asilomar Conference on Signals, Systems \& Computers, vol. 2, pp. 1655 - 1659, Nov. 1998.

[6] W. S. Lu and A. Antoniou, "Design of Digital Filters and Filter Banks by Optimization: A State of the Art Review," in Proc. EUSIPCO'2000, Tampere, Finland, Sep. 2000.

[7] R. Bregovic and T. Saramaki, "A general-purpose optimization techniques for designing two-channel FIR filter banks," in Proc. EUSIPCO' 2000, Tampere, Finland, Sep. 2000.

[8] W. Liu, S. C. Chan, and K. L. Ho, "Multiplier-less Lowdelay FIR and IIR Wavelet Filter Banks with SOPOT Coefficients," in Proc. ICASSP'2000. Istanbul, Turkey, May. 2000.

[9] S. Sriranganathan, D. R. Bull, and D. W. Redmill, "The design of low complexity two-channel lattice-structure perfect-reconstruction filter banks using genetic algorithms," in Proc. IEEE ISCAS'97, vol. 4, pp. 2393 2396, Jun. 1997.

[10] D. B. H. Tay, "Two stage, least squares design of biorthogonal filter banks," in Proc. IEEE ISCAS' 2000, pp. $591-594$ vol.1, 2000.

[11] S. C. Chan, W. Liu and K. L. Ho, "Perfect reconstruction modulated filter banks with sum of powers-of-two coefficients," in Proc. IEEE ISCAS' 2000, vol. 2, pp. 73$76,2000$. 
[12] F. Alizadeh, "Interior point methods in semidefinite programming with applications to combinatorial optimization," SIAM J. Optim., 5:13-51, 1995

[13] Y. Nesterov and A. Nemirovskii, Interior Point Polynomial Methods in Convex Programming, SIAM, Philadelphia, 1994.

[14] L. Vandenberghe and S. Boyd, "Semidefinite Programming," SIAM J. Review, vol. 38, pp. 49-95. Mar. 1996.

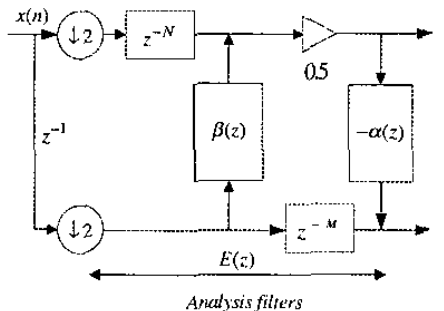

Figure 1. Structurally PR FB

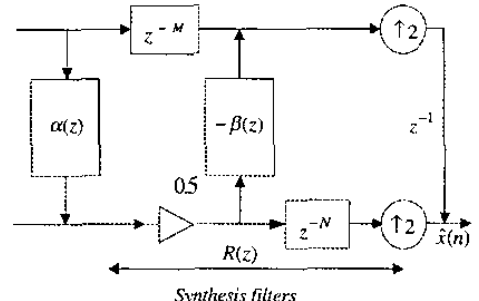

Synthesis fitters
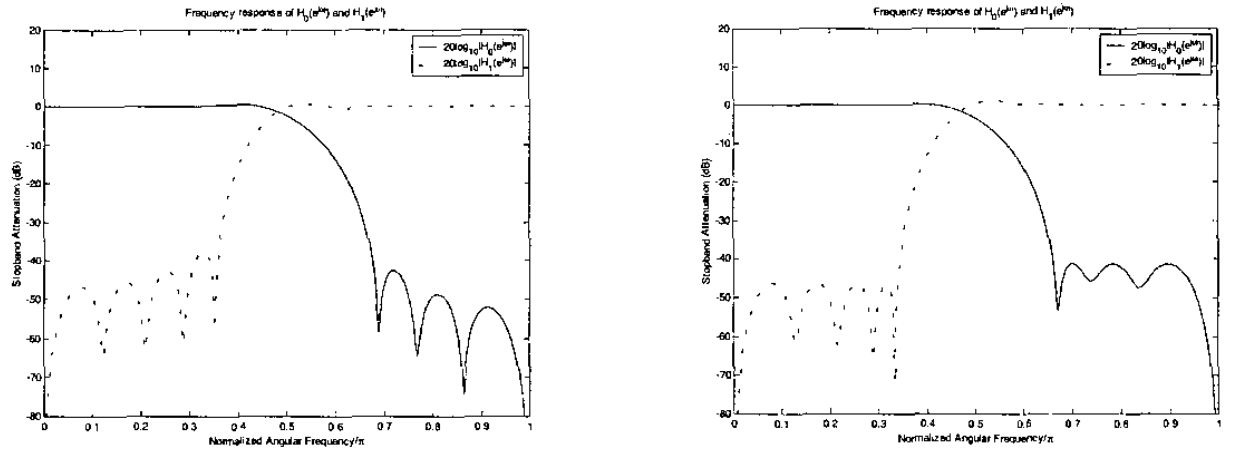

Figure (2a)

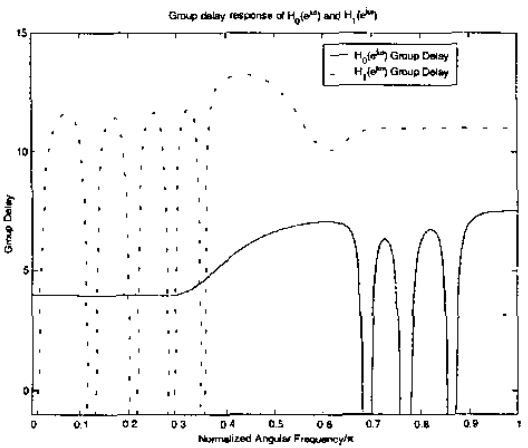

Figure (2b)

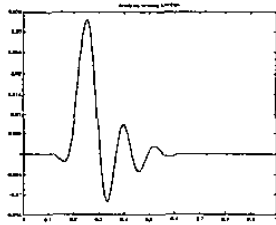

Figure (2c)

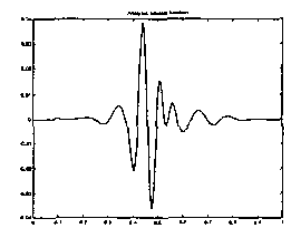

Figure (2d)
Figure 2. Design results using QPLC. (a) Frequency response of the analysis filters pair. (b) Group delay of the analysis filters pair. (c) Analysis scaling function. (d) Analysis wavelet function
[15] H. Wolkowicz, R. Saigal and L. Vandenberghe, Handbook of Semidefinite Programming - Theory, Algorithnts, and Applications, Boston: Kluwer Academic Publishers, 2000.

[16] R. Fletcher, Practical Methods of Optimization, $2^{\text {nd }}$ Edition, Chichester: John Wiley \& Sons, 1987. 